\title{
Simulation of physics background in Super c-tau factory detector
}

\author{
$L$ Shekhtman ${ }^{1,2, *}, F$ Ignatov $^{1,2}$, and $V$ Tayursky $^{1,2}$ \\ ${ }^{1}$ Budker Institute of Nuclear Physics, 11 av.Lavrentiev, 630090, Novosibirsk, Russia \\ ${ }^{2}$ Novosibirsk State University, 1 Pirogova str., 630090, Novosibirsk, Russia
}

\begin{abstract}
Simulation of background particle fluxes generated by colliding beams is performed with FLUKA package for the Super C-Tau factory Detector (SCTD). Two processes are considered as main sources of luminosity generated background: two-photon production of electron-positron pairs and Bha-Bha scattering with bremsstrahlung photon emission (radiative Bha-Bha). The SCTD geometry is described corresponding to the last version of the Conceptual Design Report. The magnetic field based on the calculation in ANSYS is introduced in the model. Main results of the simulation for beam energy of $3 \mathrm{GeV}$, luminosity of $10^{35} \mathrm{~cm}^{-2} \mathrm{~s}^{-1}$ and $1.5 \mathrm{~T}$ magnetic field are the following: charged particle fluence in the region of the Inner Tracker (radius $5 \mathrm{~cm}$ $20 \mathrm{~cm}, \mathrm{Z}$ between $-30 \mathrm{~cm}$ and $30 \mathrm{~cm})$ is between $10^{5}$ particles $/\left(\mathrm{cm}^{2} \mathrm{~s}\right)$ and $\sim 10^{3}$ particles/( $\left.\mathrm{cm}^{2} \mathrm{~s}\right) ; 1-\mathrm{MeV}$ neutron equivalent fluence for $\mathrm{Si}$ in the regions corresponding to electronics of the Inner Tracker and the Drift Chamber is below $10^{11} \mathrm{n} /\left(\mathrm{cm}^{2} \mathrm{y}\right)$ and absorbed dose is below $100 \mathrm{~Gy} / \mathrm{y}$ in the hottest regions of the detector.
\end{abstract}

\section{Introduction}

Super C- $\tau$ factory is electron-positron collider with "Crab-Waist" collision scheme, that will operate in the energy range of $1.5-3(3.5) \mathrm{GeV}$ per beam, provide luminosity up to $10^{35}$ $\mathrm{cm}^{-2} \mathrm{~s}^{-1}$ and longitudinal polarization of electrons in the interaction point [1].

The physics program of experiments at the Super c- $\tau$ factory is dedicated to studies of rare decays of D mesons, $\tau$ lepton, $D_{0} \bar{D}_{0}$ oscillations and searches for so-far unobserved lepton-flavor-violating $\tau$ decays, in particular $\tau \rightarrow \mu \gamma$ decay. The proposed program requires creation of a general purpose magnetic detector with a field of about 1.5 T. The detector should provide the following features:

- Good energy and momentum resolution

- High efficiency for soft hadrons

- Excellent $\pi / \mathrm{K}$ and $\pi / \mu$ separation

- Maximum output event rate up to $300 \mathrm{kHz}$

- Minimum CP-asymmetry of the detector

\footnotetext{
*e-mail: L.I.Shekhtman@inp.nsk.su
} 
Detector for Super c- $\tau$ factory (SCTD) consists of the Inner Tracker (IT), the Drift chamber (DC), the Particle identification system (PID), the Calorimeter, the Superconductive coil and the iron yoke with the Muon system. Conceptual design of the SCTD is shown in Fig. 1.

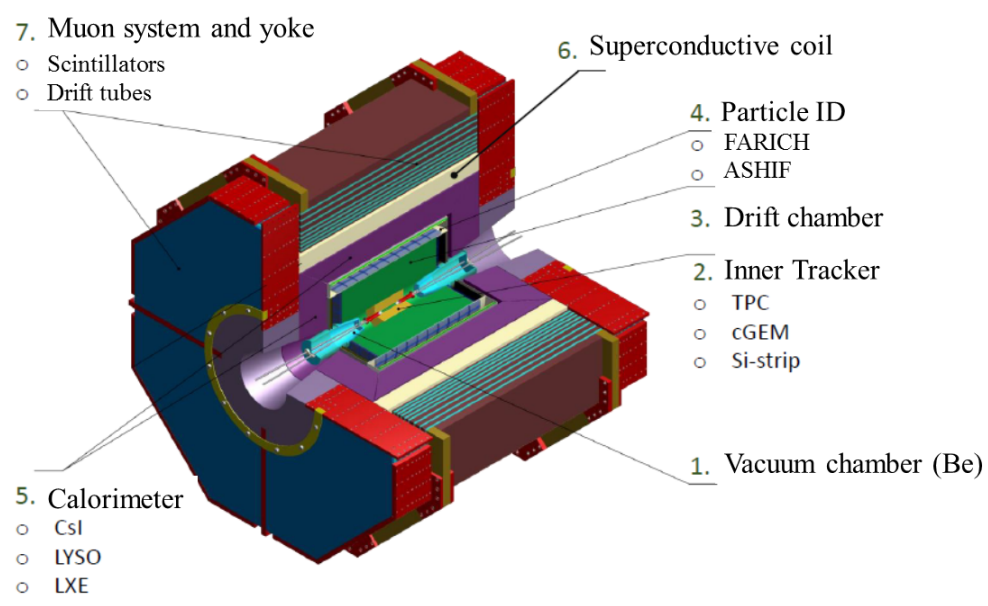

Figure 1. Conceptual design of the Super C- $\tau$ factory detector.

Most of SCTD subsystems have several options. In particular the IT has three main options: compact time projection chamber (TPC), cylindrical Gas Electron Multiplier tracker (CGEM) and silicon microstrip tracker (Si-strip). Besides that two wire options are also considered: straw tube tracker (STT) and compact drift chamber (CDC). As the IT is the closest subsystem to the interaction point, the flux of background particles is an important issue that will affect the choice of IT technology. In the present work the simulations of physics background generated by the colliding beams are performed and main consequences for all the listed options of the IT are analysed as well as distributions of particle flux, radiation dose and $1-\mathrm{MeV}$ neutron equivalent fluence for the whole SCTD.

\section{Simulations of background particle flux}

Simulations of background particle fluxes were performed with FLUKA package [2, 3]. Main sources of background generated by colliding beams are two-photon interaction of electron and positron $e^{+} e^{-} \rightarrow \gamma^{*} \gamma^{*} \rightarrow e^{+} e^{-} e^{+} e^{-}$and radiative Bha-Bha scattering $e^{+} e^{-} \rightarrow$ $e^{+} e^{-} \gamma(n \gamma)$. Particles for the first process were generated with "diag36" generator $[4,5]$ and for $3 \mathrm{GeV}$ electron and positron beams total cross-section of this process was $\sim 6 \mathrm{mb}$. Radiative Bha-Bha events were generated with "BHWIDE" generator [6]. Total cross-section with $\theta>5 \mathrm{mrad}$ and photon energy $\mathrm{E}_{\gamma}>3 \mathrm{MeV}$ was $\sim 1.7 \mathrm{mb}$. Total cross-section for two background processes is equal to $7.7 \mathrm{mb}$ that for luminosity of $10^{35} \mathrm{~cm}^{-2} \mathrm{~s}^{-1}$ gives $\sim 8 \times 10^{8}$ background events per second or eight background particles per collision ( $5 \mathrm{~ns}$ between bunches, 2 particles per event).

Two samples of particles, one for each process, were generated, mixed in proportions corresponding to the ratio of cross-sections and randomized. The final sample prepared in such way, consisting of $10^{6}$ particles, was used for the simulations. 
Schematic view of the SCTD model used for simulations is shown in Fig. 2. The model has axial symmetry, all dimensions and materials are realized according to the Conceptual Design Report [1].

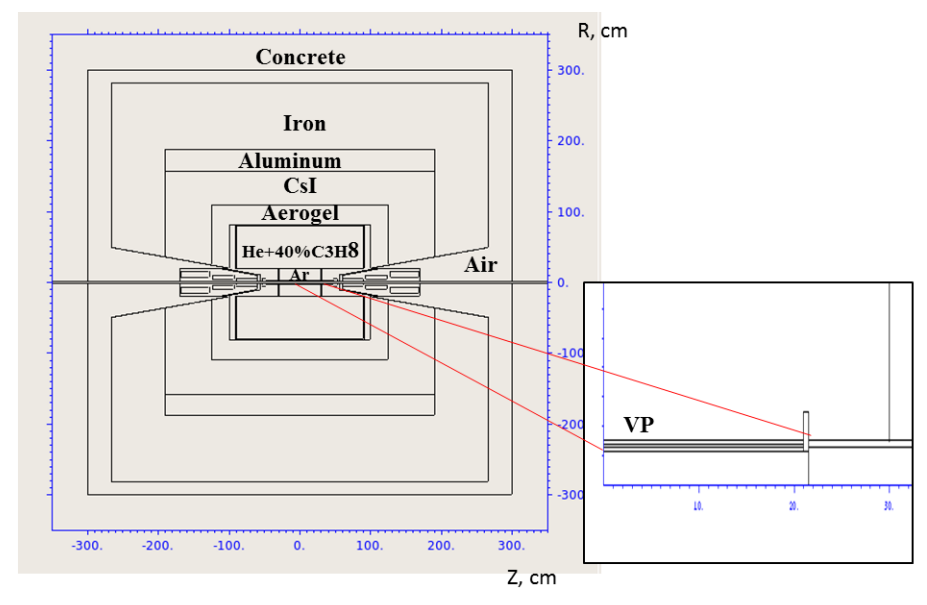

Figure 2. SCTD model in FLUKA.

The vacuum pipe in the region of the IT is realized of two nested Be cylinders, the inner one having $30 \mathrm{~mm}$ inner diameter and $1.5 \mathrm{~mm}$ wall thickness and the outer one having 34 $\mathrm{mm}$ inner diameter and also $1.5 \mathrm{~mm}$ wall thickness. The gap of $0.5 \mathrm{~mm}$ between these cylinders is filled with paraffin. The rest of the vacuum pipe is realised of $30 \mathrm{~mm}$ diameter stainless steel pipe with $3 \mathrm{~mm}$ thick wall. The IT occupies volume from $30 \mathrm{~mm}$ to $200 \mathrm{~mm}$ in radius and from $-300 \mathrm{~mm}$ to $300 \mathrm{~mm}$ in $\mathrm{Z}$ (along beam axis). In most of the simulations the TPC option of IT was realized with $1 \mathrm{~mm}$ thick walls made of glass fiber (G10) coated with $15 \mu \mathrm{m}$ of copper and all the inner volume filled with argon gas at $1 \mathrm{~atm}$ pressure. At the right and the left sides of the IT the models of the final focus magnets were realised. They are built as copper cylinders with gradually increasing radius from $5 \mathrm{~cm}$ to $20 \mathrm{~cm}$ and with increasing thickness from $2 \mathrm{~cm}$ to $10 \mathrm{~cm}$. These copper cylinders are enclosed in stainless steel containers with $10 \mathrm{~mm}$ thick walls with vacuum inside. All other parts of the model are filled with uniform materials (see Fig. 2). The whole model is surrounded with concrete walls at $\mathrm{Z}$ and $\mathrm{R}$ equal to $\pm 300 \mathrm{~cm}$.

Magnetic field was calculated with ANSYS program [7] and incorporated into the simulation. The absolute value of magnetic field within the whole tracking system is about 1.5 $\mathrm{T}$.

\subsection{Results of the simulations}

The R-Z map of electron-positron fluence in SCTD is shown in Fig. 3. Content of each bin of the map represents a total track length crossing the bin, normalized over the bin volume. Each bin is a section of pipe with $\Delta \mathrm{R}=\Delta \mathrm{Z}=1 \mathrm{~cm}$. In the region of tracking system electrons and positrons are mainly generated in the interaction point. Secondary particles are produced at the walls of the vacuum pipe by high energy electrons at small $\theta$ and backscattered into the cenral region of SCTD. Charged particle rates were simulated with different options of the IT and due to small amount of material (less than 5\% of radiation length) no significant 
difference was observed. Electron-positron fluence in the region of IT is changing from $10^{5}$ particles $/ \mathrm{cm}^{2} \mathrm{~s}$ at the inner radius to $10^{3}$ particles $/ \mathrm{cm}^{2} \mathrm{~s}$ at the outer radius.

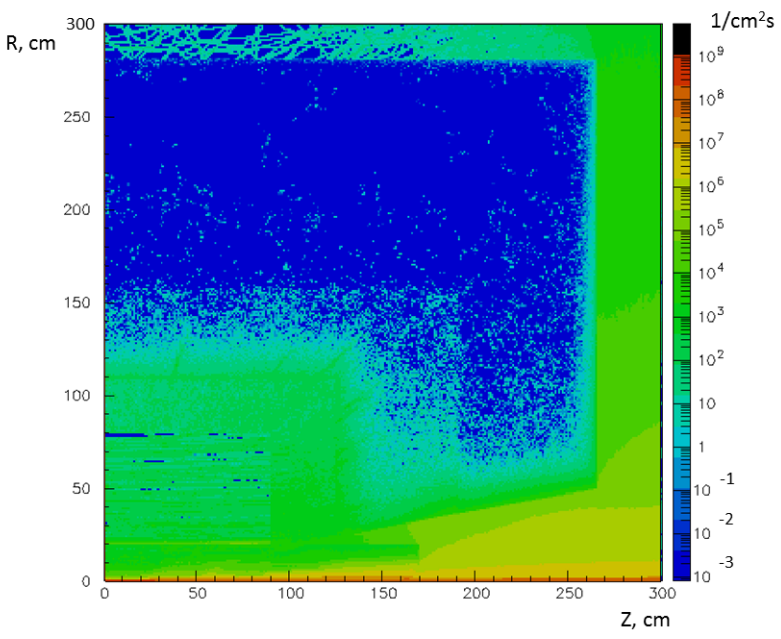

Figure 3. Fluence of electrons and positrons.

The R-Z map of 1-MeV neutron equivalent fluence for $\mathrm{Si}$ is shown in Fig. 4. Neutrons are generated by high energy photons through photo-nuclear reactions in a regions with high flux of high energy electrons and photons (final focus magnets, walls of vacuum pipe). In the regions of electronics of the IT and the $\mathrm{DC}(\mathrm{Z}= \pm 30 \mathrm{~cm}, 3 \mathrm{~cm}<\mathrm{R}<20 \mathrm{~cm}$ and $\mathrm{Z}= \pm 90 \mathrm{~cm}, 20$ $\mathrm{cm}<\mathrm{R}<100 \mathrm{~cm}$ ) $1-\mathrm{MeV}$ neutron equivalent fluence for Si does not exceed $10^{11} \mathrm{n} / \mathrm{cm}^{2}$ year and $10^{10} \mathrm{n} / \mathrm{cm}^{2}$ year respectively, assuming $10^{7}$ seconds per year of machine operation.

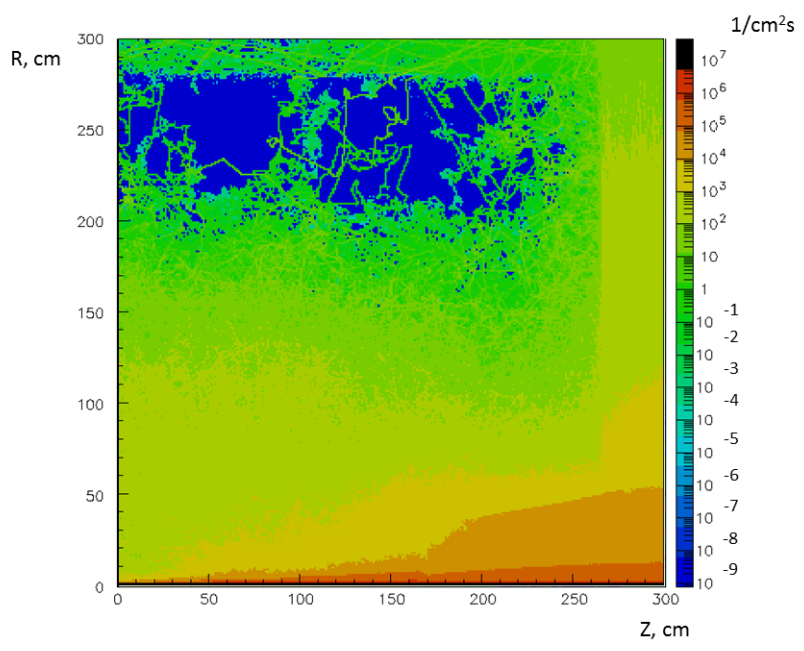

Figure 4. . 1-MeV neutron equivalent fluence for $\mathrm{Si}$ in particles $/ \mathrm{cm}^{2} \mathrm{~s}$. 
The map of absorbed dose is presented in Fig. 5. Dose is deposited mostly by secondary electrons, positrons and photons scattered from the walls of vacuum pipe and final focuse magnets. In very inner layers of the tracking system the dose is deposited by intense flux of electrons and positrons generated by two-photon interactions of primary beams. In the region of electronics of the IT absorbed dose does not exceed $100 \mathrm{~Gy} /$ year while in all other regions of the SCTD it does not exceed $10 \mathrm{~Gy} / \mathrm{year}$.

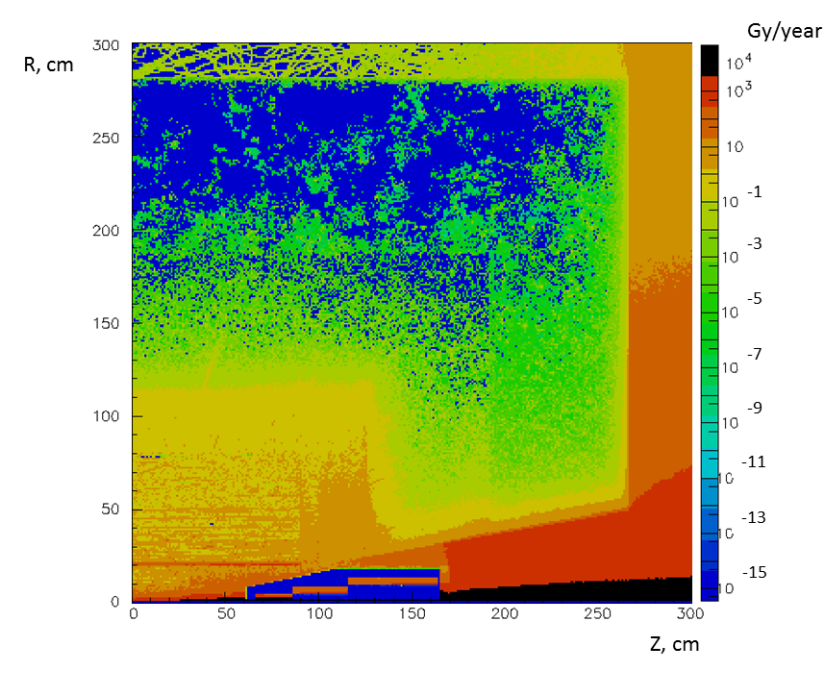

Figure 5. Absorbed dose in Gy/year.

Taking into account particle fluxes in the IT listed above, $10^{3}-10^{5}$ particles $/ \mathrm{cm}^{2} \mathrm{~s}$, channel area and dead time of each IT option, corresponding occupancy values were estimated (see Table 1). From the table one can see that occupancies of the wire options are too high while for the rest three options occupancies are quite acceptable.

Table 1. Occupancy (fraction of time, when system is busy) for different options of the Inner Tracker.

\begin{tabular}{llll}
\hline option & Channel size & Time of the measurement & Occupancy \\
\hline TPC & $1 \times 300 \mathrm{~mm}^{2}$ & $100 \mathrm{~ns}$ & $3 \times 10^{-2}-3 \times 10^{-4}$ \\
Si-strip & $300 \times 0.2 \mathrm{~mm}^{2}$ & $10 \mathrm{~ns}$ & $10^{-3}-10^{-5}$ \\
CGEM & $300 \times 0.4 \mathrm{~mm}^{2}$ & $100 \mathrm{~ns}$ & $10^{-2}-10^{-4}$ \\
Straw tubes & $600 \times 10 \mathrm{~mm}^{2}$ & $100 \mathrm{~ns}$ & $1-0.01$ \\
Compact drift chamber & $600 \times 5 \mathrm{~mm}^{2}$ & $100 \mathrm{~ns}$ & $0.5-0.005$ \\
\hline
\end{tabular}

\section{Summary}

The first simulations of the background particle fluxes generated by colliding beams were performed with FLUKA package. The SCTD model used in the simulations has axial symmetry and includes straight vacuum pipe.Fluence of electrons and positrons, that is responsible for occupancy in the tracking system, does not exceed $10^{5}$ particles $/ \mathrm{cm}^{2} \mathrm{~s}$ at the inner radius of the Inner Tracker and drops to $10^{3}$ particles $/ \mathrm{cm}^{2} \mathrm{~s}$ at the outer radius of the IT. $1-\mathrm{MeV}$ neutron equivalent fluence for Si and absorbed dose do not exceed $10^{11} \mathrm{n} / \mathrm{cm}^{2}$ year and $100 \mathrm{~Gy} /$ year 
respectively in the regions of electronics of the tracking system. Occupancies of different options of the IT were estimated using the values of particle fluence, and it appeared that wire options, such as straw tubes and compact drift chamber can not be used in such environment.

\section{References}

[1] https://ctd.inp.nsk.su/c-tau/

[2] T.T Bohlen et al., The FLUKA Code: Developments and Challenges for High Energy and Medical Applications, Nuclear Data Sheets, 120, 211-214 (2014).

[3] G. Battistoni et al., Overview of the FLUKA code, Annals of Nuclear Energy 82, 1018(2015).

[4] Frits A. Berends, P.H. Daverveldt, R. Kleiss, Monte Carlo Simulation of Two Photon Processes. 2. Complete Lowest Order Calculations for Four Lepton Production Processes in electron Positron Collisions, Comput.Phys.Commun. 40 (1986) 285-307

[5] Frits A. Berends, P.H. Daverveldt, R. Kleiss, Complete Lowest Order Calculations for Four Lepton Final States in electron-Positron Collisions, Nucl.Phys. B253 (1985) 441463.

[6] S.Jadach et.al., in Physics at LEP2, edited by E.Altarelli, T.Sjostrand and F.Zwirner (CERN, Geneva, 1996), Vol.2, p.229, Yellow Report CERN 96-01, e-Print Archive: hepph/9602393.

[7] Private communication with A.Bragin. 